\title{
Study on the interpretation method of formation-elements logging
}

\author{
Weiwei He $\mathrm{He}^{1, \mathrm{a}}$, Liang Sun ${ }^{2, \mathrm{~b}}$ and Yanling $\mathrm{Liu}^{2, \mathrm{c}}$ \\ ${ }^{1}$ School of Mechatronics Engineering, Daqing Normal University, Daqing, Heilongjiang, China \\ ${ }^{2}$ Logging \& Testing Services Company, Daqing Oilfield Co. Ltd., Daqing, Heilongjiang, China \\ avivian820626@163.com, ${ }^{\mathrm{b}} \mathrm{cdlts}$ _sunliang@petrochina.com.cn, ${ }^{\mathrm{c}} \mathrm{cdlts}$ _liuyl@petrochina.com.cn
}

Keywords: formation element; standard spectrum; pulsed neutron

Abstract. With the application of the pulsed-neutron formation-elements logging instrument, the interpretation method of formation-elements logging has been proposed. We obtained the standard spectrum library for formation elements, got the element yield, dry weight(percent content of the quality without crystalline water), and the content of mineral components. The core well experiment results show that the interpretation results are in good agreement with the core test results.

\section{Introduction}

In order to calculate the density of igneous rock skeleton, to identify the lithology of carbonate profilethe, and to accurately calculate the porosity, the elemental capture spectroscopy logging technology must be adopted in the reservoir evaluation of the deep gas and complex lithologic area.

At present, there are three instruments for identifying formation lithology by collecting captured gamma energy spectrum to measure element content. They are formation-elements energy-spectrum logging instrument(ECS) of Schlumberger, geochemical logging instrument of neutron-gamma spectroscopy (GEM) of Halliburton, and pulse-neutron stratigraphic-lithologic energy spectrometer(FLeX) of Baker Hughs. In 2012, Based on the pulsed neutron technique, the formation-elements analysis instrument: lithologic scanner(Litho Scanner) is introduced by Schlumberger. Pulse neutron source, $\mathrm{LaBr} 3: \mathrm{Ce}$ detector and the acquisition and processing technologies of high-speed digital energy spectrum are used in this instrument. With a high resolution, this instrument can provide the contents of 16 elements and total organic carbon of the stratum. It is known as the most advanced analytical instrument of the formation elements in the world ${ }^{[1]}$.

Based on MCNP numerical simulation method, the standard spectrum library of formation elements was obtained, the interpretation method was developed, and the coring well experiment was carried out to verify the method.

\section{The principle of formation-elements logging}

As shown in Fig. 1, after the fast neutron from the neutron generator with $14.1 \mathrm{MeV}$ energy into the stratum, the inelastic scattering first occurs. After about $10^{-8} \mathrm{~s} \sim 10^{-7} \mathrm{~s}$, inelastic gamma ray comes into being. Then after elastic scattering about $10^{-6} \mathrm{~s} \sim 10^{-3} \mathrm{~s}$, almost all fast neutrons are slowed to thermal neutrons. They are always captured by the surrounding media, and release the capture gamma ray.The final gamma ray produced from the decay of active nucleus ${ }^{[2]}$.

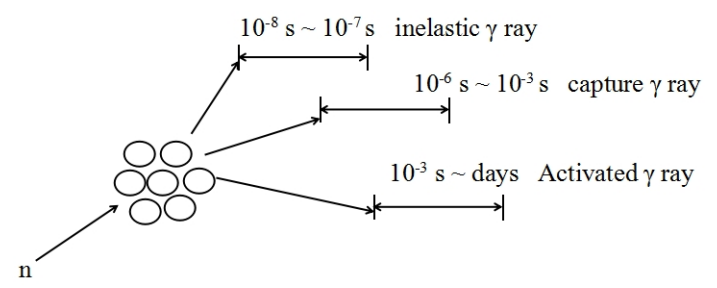

Fig. 1. Time distributions of several gamma rays induced by neutrons 
After the fast neutron enter into the stratum, the capture gamma ray is released. We can collect capture spectrum of the characteristic elements, so the relative yield of characteristic elements can be obtained by solving the spectrum. Then the relative percent of the formation elements is obtained from the oxygen closure model. Also, we can analyze the relative percentage of the formation elements by establishing the related interpretation model, and can get lithology, porosity, skeleton density and other related geological parameters.

\section{The interpretation method of formation-elements logging and the software realization}

The model of MCNP numerical simulation was set up which was based on building program of simulation well of formation element. We contrasted the results of simulation with the results of instrument's measure in simulation well. By modying the uncertain material in simulated model, the simulation results were consistent with the experimental results. Finally, we can confirm that the model is reasonable.

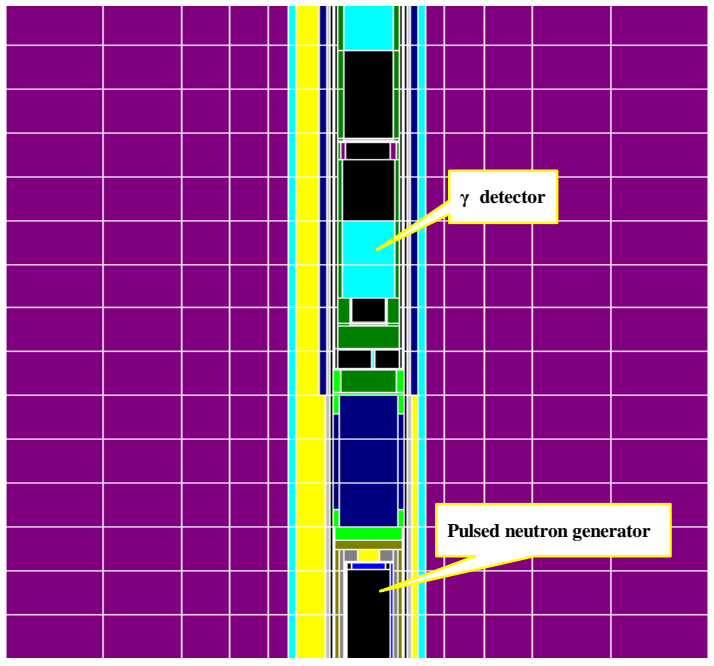

Fig. 2. Collection model of formation-element standard energy-spectrum

As shown in Fig.2, the numerical-simulation model of elemental standard energy-spectrum was set up which was based in Reference checking of numerical simulation. By using numerical simulation method, we build element standard energy-spectrum with $\mathrm{Si}, \mathrm{Ca}, \mathrm{S}, \mathrm{Fe}, \mathrm{Ti}, \mathrm{Gd}, \mathrm{H}, \mathrm{Cl}, \mathrm{K}$, $\mathrm{Na}, \mathrm{Mn}, \mathrm{Mg}$ under different temperature and density, as shown in Fig. 3. All that in diffrent temperature and density by numerical simulation.

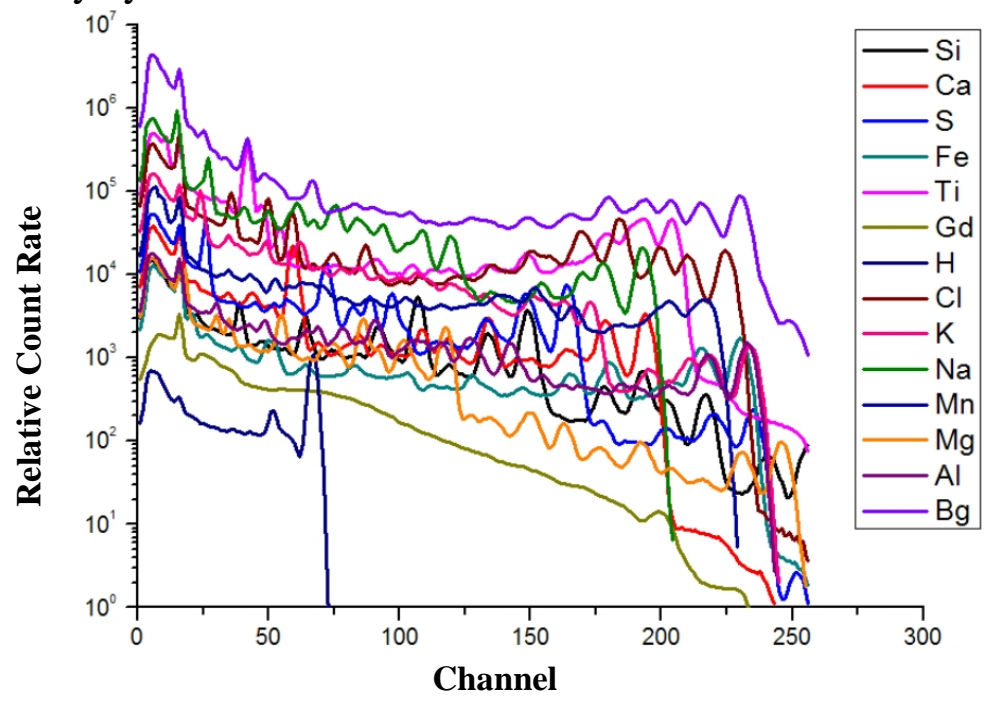

Fig. 3. Standard trapping energy spectrum of formation element 
The process of standard spectrum acquisition is as follows:

(1) Based on the perfect numerical simulation model, the gamma-flux counting of the detector surface;

(2) Calculate the logging response matrix of the detector;

(3) Get the standard spectrum of different element by calculating the convolution of detector surface flow count and the logging response matrix;

(4) Energy calibration and peak broadening correction for standard energy spectra.

The energy of gamma ray is different when the capture nuclear reaction happened from thermal neutron and different formation element nucleus. By using BGO crystal detector to record gamma spectrum, we can qualitative and quantitative confirm the element and the content. The content of the above is the logging method of formation-elements trapped energy-spectrum.

The core formula for the content of elements which were measured by formation-elements logging instrument is ${ }^{[3]}$ :

$$
W_{t j}=F \frac{y_{j}}{S_{j}}
$$

$W_{t j}$ is the weight percentage of the $\mathrm{j}$-th element in the ground. $\mathrm{y}_{\mathrm{j}}$ is the yield of the $\mathrm{j}$-th element. It is the contribution of the neutron capture gamma-spectra of the j-th element to the mixed spectra. $S_{j}$ is relativly sensitivity factor of the $\mathrm{j}$-th element. It is related with the element and detector, and without the intensity of the neutron source, the neutron transport, and the formation density. $F$ is the normalized factor. it is only related with the instrument and the groud where the instrument is, so it changes with the groud changing.

As the theory evidence of closed normalization model, for a certain stratum, the sum of all the weight percentage should be 1 . The formula 1 can be changed as follows if oxide and carbonate minerals are the main components in the formation,

$$
F \sum_{j=1}^{m} X_{j} \frac{y_{j}}{S_{j}}=1
$$

In this case, $X_{j}$ is used as the oxide index of the $\mathrm{j}$-th oxidizing materials and carbonate minerals in this normalization model.

$$
F=1 / \sum_{j=1}^{m} X_{j} \frac{y_{j}}{S_{j}}
$$

$y_{j}$ can be obtained by explaining the measured formation spectrum, $S_{j}$ can be obtained from the scale of the model well, $F$ can be obtained from the oxide closure model, then we can get the percentage of the element.

If the component of the mineral remains stable, by using multiple regression analysis, we can get the relation between the content of the formation-elements and the mineral.

$$
[M]=[C]^{-1}[E]
$$

In this case, $[M]$ is the percentage of the mineral, $[E]$ is the percentage of the formation-elements, $[C]$ is the coefficient matrix. Matrix element $\mathrm{Cij}$ of which can be found in the literature, or be obtained by regression analysis.

\section{Field Application}

L-J\# is the well of Daqing oilfield. Field experiment was carried out at L-J\# in a open hole well, which is a core well. The measuring range of the well was $950 \sim 1000 \mathrm{~m}$. The first measuring was $950 \sim 1050 \mathrm{~m}$. The measurement speed was $60 \sim 70 \mathrm{~m} / \mathrm{h}$. 


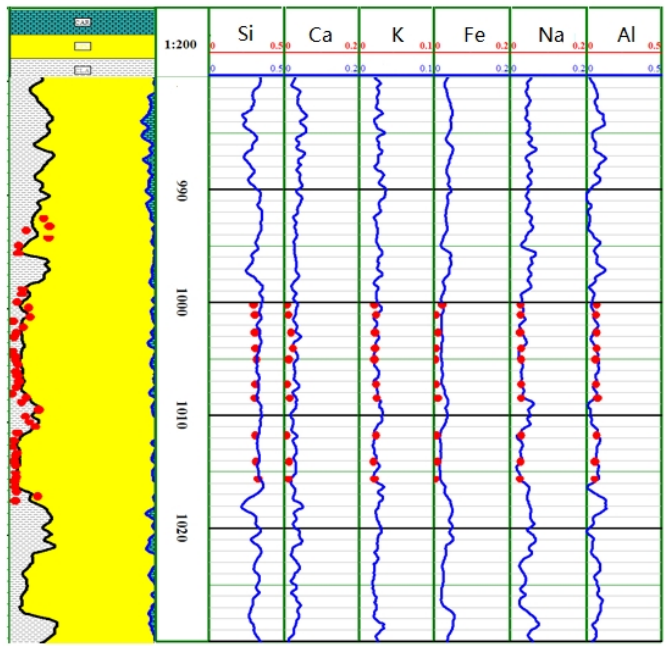

Fig. 4. Logging interpretation results for L-J\#

As shown in Fig. 4, the red points are the results of coring, the blue lines are the mineral weight, they fit very well. The maximum absolute error of calculating $\mathrm{CaO}, \mathrm{K} 2 \mathrm{O}, \mathrm{Si}_{2} \mathrm{O}, \mathrm{Al}_{2} \mathrm{O}_{3}, \mathrm{Na}_{2} \mathrm{O}, \mathrm{Fe}_{2} \mathrm{O}_{3}$ is $3.13 \%, 0.43 \%, 3.07 \%$., $2.21 \%, 0.65 \%, 1.99 \%$, respectively.

\section{Conclusions}

Based on the principle of neutron-gamma reaction, the interpretation method of formation-elements trapping energy-spectrum was studied. and the model of formation-elements logging interpretation has been built. We got the formation-element trapping energy-spectrum by combining with numerical simulation and the measurement of the standard well. The comparison with the coring data of open hole well and interpretation results of Litho Scanner show that, the interpretation method of formation-elements logging can get the accurate interpretation results.

\section{Acknowledgements}

This work was financially supported by the Science Research Fund of Daqing Normal University (grant No. 10ZR14).

\section{References}

[1] Chengfang Yin, Shizhen Ke. World Well Logging Technology. Vol. 192 (2012), p. 73. In Chinese.

[2] Longji Hang: Radioactive Logging Principle (Petroleum Industry Press, Beijing 1985). In Chinese.

[3] Jufeng Long. Journal Of Isotopes. Vol. 12 (2006), p. 70. In Chinese. 